This article was downloaded by: [University of Waterloo]

On: 07 February 2012, At: 05:45

Publisher: Routledge

Informa Ltd Registered in England and Wales Registered Number:

1072954 Registered office: Mortimer House, 37-41 Mortimer

Street, London W1T 3J H, UK

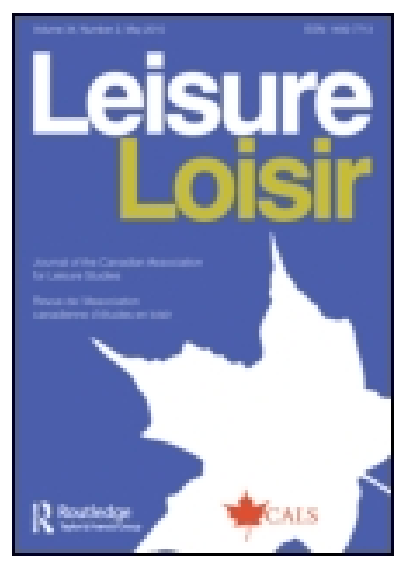

\title{
Leisure/Loisir
}

Publication details, including instructions for authors and subscription information: http:// www. tandfonline.com/loi/ rloi20

\section{Points de repère sur la pensée spirituelle de Robert Stevenson Smyth Baden-Powell}

\begin{abstract}
Gervais Deschênes ${ }^{a}$
${ }^{a}$ Chargé de cours au département des sciences de l'éducation et de psychologie, Université du Québec à Chicoutimi, 555, boulevard de I'Université, Chicoutimi, Québec, G7H 2B1 Phone: (418) 276-7443 E-mail:
\end{abstract}

Available online: 21 Nov 2010

To cite this article: Gervais Deschênes (2009): Points de repère sur la pensée spirituelle de Robert Stevenson Smyth Baden-Powell, Leisure/ Loisir, 33: $1,55-77$

To link to this article: http:// dx. doi. org/ 10.1080/ 14927713.2009.9651430

PLEASE SCROLL DOWN FOR ARTICLE

Full terms and conditions of use: http://www.tandfonline.com/ page/terms-and-conditions

This article may be used for research, teaching, and private study purposes. Any substantial or systematic reproduction, 
redistribution, reselling, loan, sub-licensing, systematic supply, or distribution in any form to anyone is expressly forbidden.

The publisher does not give any warranty express or implied or make any representation that the contents will be complete or accurate or up to date. The accuracy of any instructions, formulae, and drug doses should be independently verified with primary sources. The publisher shall not be liable for any loss, actions, claims, proceedings, demand, or costs or damages whatsoever or howsoever caused arising directly or indirectly in connection with or arising out of the use of this material. 


\title{
Points de repère sur la pensée spirituelle de Robert Stevenson Smyth Baden-Powell
}

\author{
Gervais Deschênes \\ Université du Québec à Chicoutimi
}

Résumé. Cet article s'inscrit dans le cadre d'un débat à l'Association des Scouts du Canada. Ainsi, devant le pluralisme religieux de la société canadienne, le scoutisme francophone est appelé à développer sa dimension religieuse. Dans la poursuite de la réflexion, il est nécessaire de retoumer à l'oeuvre littéraire du fondateur du mouvement, Robert Stevenson Smyth Baden-Powell et de constater l'aspect avant-gardiste de sa pensée spirituelle au début du XXe siècle. À partir de ses expériences de jeunesse et militaire, Baden-Powell développe un mouvement multiconfessionnel qui se distingue du conservatisme religieux de son époque. En ce sens, sa pensée est le premier jalon pour une spiritualité du jeu qu'il met en pratique en faveur de la jeunesse mondiale tout en respectant les différentes confessionnalités. La spiritualité du jeu inhérente au scoutisme constitue un modèle quant à une compréhension mutuelle et au dialogue moral dars une perspective multiculturelle.

Mots-clés. Baden-Powell, scoutisme, spiritualité, jeu

Abstract. This study focuses on a discussion within the Association des Scouts du Canada. Faced with religious pluralism in Canadian society, francophone scoutism is called upon to construct its religious identity. To investigate this issue, it is necessary to go back to the writing of the founder of scoutism, Robert Stevenson Smyth Baden-Powell, and to consider his avant-gardist spiritual vision at the beginning of the twentieth century. From his youth and military experiences, Baden-Powell developed a multidenominational movement which distanced itself from the religious conservatism at this time. In this sense, his thoughts are the first step toward a spirituality of play for international youth that respects the different aspects of other people's religions. Baden-Powell's spirituality of play provides a model for a mutual understanding and moral guidance from a multicultural perspective.

Keywords. Baden-Powell, scoutism, spirituality, play

Address all correspondence to: Gervais Deschenes, Chargé de cours au département des sciences de l'éducation et de psychologie, l'Université du Québec à Chicoutimi, 555, boulevard de 1'Université, Chicoutimi, (Québec) G7H 2B1. Téléphone: (418) 2767443. Courriel: gervais_deschenes@uqac.ca 
« Joue ne te borne pas à regarder ». —-Baden-Powell

\section{Genèse de la réflexion}

Le scoutisme francophone est un exemple d'une communauté organisée qui aide les jeunes Canadiens à poursuivre leur développement spirituel dans un contexte de jeu/loisir (Gravelle \& Deschênes, 2004). Les relations dynamiques entre les jeunes par le jeu représentent des temps forts au sein du scoutisme qui se distingue des autres organismes bénévoles par son identité religieuse toujours en questionnement face aux différentes valeurs sociales en changement. Le scoutisme est un mouvement d'éducation; une école de vie qui s'inscrit dans une pédagogie permettant aux scouts de vivre un développement progressif de leur personnalité aux plans physique, intellectuel, social et spirituel (Organisation Mondiale du Mouvement Scout [OMMS], 1992). Au plan international, le scoutisme est un mouvement apolitique ouvert à tous sans distinctions de races, de religions ou de croyances. De fait, il regroupe plusieurs appartenances religieuses et laïques qui cohabitent ensemble.

L'Association des Scouts du Canada reflète son contexte socioculturel bien particulier de sorte qu'il n'est pas identique aux autres organisations scoutes à travers le monde. Elle possède une structure de fonctionnement qui lui permet de privilégier la transmission de valeurs humaines et chrétiennes. Elle permet aussi d'échanger des opinions entre les membres qui font face aux phénomènes $d$ 'incroyance, $d$ 'indifférence et de pluralisme religieux à l'heure actuelle. Or, le scoutisme francophone canadien subit présentement une décroissance due à un problème d'image qui, pour certains adultes éducateurs, est relié à son appartenance religieuse catholique. Ainsi, le scoutisme francophone canadien est profondément ancré à sa symbolique religieuse (croix de Jérusalem, chants religieux, rites d'engagement, etc.). Toutefois, il est en quête d'identité permanente dans une prise de conscience qui s'articule autour des débats animés lors des rencontres nationales menées par les principaux leaders qui sont à toute fin utile laïcs. Ceux-ci se posent la question de la place du religieux dans le scoutisme.

Pour certains adultes éducateurs, il faudrait laïciser davantage le scoutisme francophone canadien dans sa présentation c'est-à-dire écarter tout esprit confessionnel puisque la société canadienne est en constante évolution et celle-ci s'exprime par son caractère pluriethnique et multiculturel. À titre d'exemple, le débat social depuis la Révolution tranquille au Québec sur le déplacement du religieux qui a résulté à la désaffectation de la pratique religieuse, la déconfessionalisation des 
écoles publiques et les discussions animées sur les accommodements raisonnables démontrent du processus de sécularisation c'est-à-dire que les « secteurs de la société et de la culture sont soustraits à l'autorité des institutions et des symboles religieux » (Berger, 1971, p. 174). Ces adultes éducateurs cherchent à revitaliser le scoutisme francophone canadien par une symbolique plus neutre et, selon eux, plus acceptable pour les jeunes.

Cette dernière position s'oppose à la vision des adultes éducateurs qui militent pour le statu quo c'est-à-dire un mouvement qui se veut un " modeste ferment de témoignage chrétien en un monde postchrétien " (Savard, 1994, p. 232). Ils ne veulent pas oublier de reconnaître les bienfaits de ce mouvement qui a permis de construire leur identité. Pour eux, il demeure important de transmettre l'héritage religieux et la mémoire historique de la tradition catholique sans pour autant être nécessairement sous la tutelle des institutions. Ils suggèrent plutôt la responsabilisation des adultes éducateurs en matière religieuse. C'était d'ailleurs dans cet esprit que s'était réalisé en 1999 le changement du terme « pastorale » pour l'expression « développement spirituel » pour l'adulte éducateur responsable des affaires religieuses. Ce nouveau vocable interpelle la libre pensée parce qu' « il n'est pas important que tous pensent la même chose. Au contraire, il est préférable que chacun puisse découvrir sa propre voie de développement spirituel » (Association des scouts du Canada [ASC], 1999, p. 11). Cette bipolarisation du débat entre adultes éducateurs n'est pas sans conséquence sur la gestion du scoutisme. On observe chez eux un certain malaise à s'entendre sur la manière de vivre la dimension religieuse. Les adultes éducateurs cherchent d'abord à définir l'espace du religieux. lls mettent des frontières et veulent subordonner celui-ci. C'est ainsi que les adultes éducateurs s'opposent ou se séparent les uns des autres et les différents acteurs sont entraînés dans un conflit territorial plus ou moins implicite.

On peut dès lors se demander jusqu'à quel point les adultes éducateurs respectent la pensée du fondateur du mouvement, Robert Stevenson Smyth Baden-Powell. Il existe donc une nécessité de retourner à l'oeuvre littéraire de Baden-Powell qui a développé une pensée sur le religieux à partir de la situation de son époque qui se répercute dans la société moderne. Mais avant toute chose une biographie du fondateur du scoutisme est ici nécessaire.

\section{Biographie du fondateur du scoutisme}

Pour mieux concevoir la pensée du fondateur du scoutisme, il importe de tracer les grandes lignes tumultueuses de sa vie bien décrite dans 
une biographie récente (Maxence, 2003). Bien saisir sa vie et sa riche personnalité c'est comprendre l'impact qu'a son mouvement dans le monde. Baden-Powell naît à Londres le 22 février 1857 d'une famille de six enfants. Son père est théologien et pasteur protestant. Il meurt trois ans après la naissance du jeune Robert. Sa mère a alors la lourde tâche d'élever seule sa famille. Grand amateur de plein air, Baden-Powell pratique le camping, les excursions, le dessin, la musique et la voile. Il s'entraîne à plusieurs sports sans toutefois y exceller. D'un tempérament enjoué, audacieux et responsable, il s'enrôle dans l'armée britannique en 1876 . Il n'a que 19 ans et se classe second en cavalerie et cinquième en infanterie sur 700 candidats. On le nomme alors officier dans le $13^{\mathrm{e}}$. Hussards (les Dragons verts). Envoyé aux Indes, il se spécialise dans la reconnaissance, le relevé topographique et le rapport en faisant l'expérience de la dure réalité militaire et coloniale (Maxence). Il devient alors un instructeur de talent grâce à son sens vif de l'observation. Il est tour à tour envoyé en mission de reconnaissance dans les Balkans, en Afrique du Sud et à Malte. En Afrique, les indigènes le surnomment « Impeesa » qui signifie « le-loup-qui-ne-dort-jamais ». En 1899, il participe à titre de commandant à la guerre des Boers où il s'illustre à la bataille de Mafeking dont le siège dure 217 jours. C'est lors de ce siège qu'il met en pratique ses techniques de renseignements grâce à une vingtaine d'adolescents de 12 à 17 ans. Éclaireurs à bicyclette, ils ont la responsabilité de la communication des informations tactiques entre les forts et la ville. Après la victoire de Mafeking en mai 1900, il devient un héros national et est nommé général à l'âge de 43 ans. De retour en Angleterre après la signature de la paix (1902), il est désigné inspecteur général de la cavalerie.

Prenant quelques libertés avec les règles militaires, il ne pourra pas être un grand général de l'armée anglaise et ses qualités de chef le mènent ailleurs. Il a voulu mettre ses compétences au service de la paix (Maxence, 2003). Baden-Powell s'aperçoit rapidement de l'engouement des jeunes pour un livre qu'il a écrit, Aids to Scouting for NCO's and men (1899). Il y enseigne l'art de l'observation et les techniques de la vie dans les bois pour les soldats. Dès lors, il commence à s'intéresser à ce phénomène et décide de mettre en pratique sa pédagogie en rassemblant une vingtaine de jeunes londoniens désoeuvrés. Ainsi, c'est du 25 juillet au 9 août 1907 que Baden-Powell et ses éclaireurs vivent le premier camp scout sur l'île de Brownsea. Devant la réussite de ce camp, il écrit un autre livre qui s'intitule Éclaireur (1908/1951) qui devient également un best-seller (Maxence). Cet ouvrage présente un projet pédago- 
gique qui propose une règle de vie inspirée de l'esprit chevaleresque fondé sur la confiance, le sens des responsabilités, l'esprit d'équipe et le jeu en plein air. Pour résumer sa méthode, Baden-Powell utilise ses propres initiales « $\mathrm{BP}$ » à la devise bien connue: « Sois prêt » (Be Prepared). Le scoutisme est né. Ce mouvement prend tellement de l'ampleur que, sur les conseils du roi Édouard VII, Baden-Powell quitte l'armée britannique en 1910 afin de consacrer tout son temps à ce mouvement de jeunesse.

En 1912, il épouse Olave Saint-Clair Soames qui fait la promotion des guides au nom de cette organisation pour jeunes filles. Ils ont trois enfants. Au cours de leur vie, le couple Baden-Powell ne cesse de faire la promotion du scoutisme et du guidisme à travers plusieurs voyages dans le monde. À titre d'exemple, Baden-Powell entreprend quatre voyages au Canada $(1910,1919,1923,1935)$. Ces voyages sont l'occasion d'être reconnu sur le plan international où il reçoit plusieurs titres honorifiques dont le «Chef scout du monde » en 1920 et le titre de noblesse de 'Lord Baden-Powell of Gilwell' en 1929. Âgé de 81 ans et malade, lui et son épouse quittent l'Angleterre pour s'établir à Nyeri au Kenya. C'est là au coeur de l'Afrique que le fondateur du scoutisme meurt le 8 janvier 1941 à l'âge de 84 ans. Il reçoit les honneurs d'une oeuvre qui n'a pas cessé de créer des liens d'amitié (Maxence, 2003). Pour sa part, Lady BadenPowell continue à développer le mouvement du scoutisme et du guidisme pour s'éteindre en 1977 dans le Surry, au sud de l'Angleterre.

Après un siècle de scoutisme et de guidisme, le mouvement se développe en gardant les principes fondamentaux élaborés par son fondateur. Ce qui sort de l'ordinaire, c'est que le scoutisme est un mouvement mondial. Depuis sa fondation, il a accueilli environ 253 millions de jeunes dans le monde. En 2006, l'Organisation mondiale du mouvement scout compte approximativement 28 millions de membres répartis dans 155 organisations nationales regroupées dans 215 pays et territoires (World Organisation of the Scout Movement [WOSM], 2006, p. 91). Plus spécifiquement dans le scoutisme francophone canadien, on dénombre 40 districts dont 20876 membres, soit 15010 jeunes (filles et garçons de 7 à 21 ans) et 5866 adultes éducateurs (ASC, 2007, p. 13).

\section{Définitions des concepts « jeu », « religion/religieux » et} « spirituel/spiritualité »

Il est ici important de donner quelques définitions opérationnelles sur les concepts « jeu », « religion/religieux » et « spirituel/spiritualité » permettant de sortir de l'impasse de la problématique du débat dans le scoutisme 
francophone canadien. Tout d'abord, le sens étymologique du mot « jeu » dérive du latin « jocus » qui signifie un amusement ou une plaisanterie plus ou moins vulgaire (Picoche, 1991). On accepte ordinairement le grec « paidia » qui va dans le sens du « jeu » en français et « das spiel » en allemand. Dans sa théorie cosmique du jeu, Fink reprend le fragment 52 d'Héraclite qui affirme que « le temps du monde est un enfant qui joue au tric-trac; son royaume est celui d'un enfant » (1966, p. 28). Le jeu est donc relié au monde de l'enfance en se différenciant du sentiment de puérilité (Huizinga, 1951). Dans cet article, nous considérons le caractère polysémique dans une perspective générale des notions de jeu, loisir et humour. L'unité de sens de ces notions doit êtré comprise comme un mode d'existence dans l'action humaine.

La recherche propose plusieurs définitions du mot « religion/religieux ». Ce concept a « subi une telle dérive de sens que les analyses que l'on poursuit sous son étiquette risquent de s'égarer » (Dumont, 1987, p. 218). Cette étude sur la spiritualité du jeu inhérente au scoutisme s'appuie sur le sens du mot « religion/religieux » présenté par Tillich qui comprend ce concept dans une approche ouverte:

Être religieux signifie s'interroger passionnément sur le sens de notre vie et être ouvert aux réponses, même si elles nous ébranlent en profondeur. Une telle conception fait de la religion quelque chose d'universellement humain, encore qu'elle s'écarte de ce qu'on entend ordinairement par religion. Celle-ci, en tant que dimension de la profondeur, n'est pas la foi à l'existence des dieux, ni même à l'existence d'un seul Dieu. Elle ne consiste pas en attitudes et en organisations où se manifeste le lien de l'homme avec son Dieu. Nul ne contestera que les religions historiques soient « religion » en ce sens. Mais une religion en sa véritable essence est plus que cela: elle s'identifie à l'être de l'homme pour tout ce qui met en cause le sens de la vie comme son existence même. (Tillich, 1969, p. 49)

La religion comprise dans ce sens suppose une dynamique du « souci ultime de l'être humain " (Tillich, 1957, p. 1) à travers une quête de foi en l'Inconditionné. Autrement dit, la foi comme "souci ultime » est un mouvement qui consiste à être saisi par une préoccupation ultime de l'humain. Celui-ci est dynamisé à aller au bout de soi-même. Cela peut l'amener alors à poser des questions à un Dieu personnel sur son rapport au monde (Tillich, 1970). Cette foi comme « souci ultime », est aussi une espérance dans la promesse qui est annoncée. Or, on oublie souvent aujourd'hui que « chaque question [possède] une force que la réponse ne [contient] plus » (Wiesel, 1958, p. 13). Ainsi, dans cette quête de 
sens et de Dieu, ce qu'il faut maintenant lui demander, ce n'est pas uniquement les bonnes réponses, mais particulièrement de « donner la force de pouvoir lui poser de vraies questions » (Wiesel, p. 14). Cette attitude de l'esprit à poser les bonnes questions fait que « la foi ne commence pas par des certitudes, mais par des questions. La foi est recherche » (Tremblay, 1997, p. 21). Une recherche qui s'incarne dans l'expérience et qui fait que Dieu demeure une question toujours ouverte.

Le mot «spirituel» correspond selon le vocabulaire biblique à 《 ruah » en hébreu, à « pneuma » en grec, et à « spiritus » en latin. Étymologiquement, le mot « spirituel » prend le sens de « respirer », " souffle vital, âme » et « de la nature de l'esprit, immatériel » (Picoche, 1991, p. 627). Dans la perspective occidentale, le spirituel renvoie à ce qui est vivant et se traduit par « l'élan vital et corporel », une « activité de la conscience » ou « les valeurs de l'esprit» (Bernard, 1986, p. 24). Nous adhérons à la définition phénoménologique de Waaijman (1993) qui soutient que « la spiritualité est la transformation continue impliquée dans la relation engagée ou engageante avec l'Inconditionnel » (p. 45). En ce sens, la spiritualité est une quête de sens continue du sujet qui attend des réponses à son questionnement. Cette définition de la spiritualité est séculière et fait partie des processus de la vie:

La spiritualité séculière est profondément liée avec le processus primordial de la vie: le processus de l'éducation et de l'apprentissage, le processus d'être chez-soi dans l'environnement qui nous entoure. Ces processus ne sont pas régulés par le religieux des institutions ou d'écoles spirituelles. Ils sont liés avec les processus primordiaux de la vie, et sont en ce sens originels et primaires. (Waaijman, 2004, p. 14)

Il y a donc des similitudes de sens entre les termes « religion/religieux » et "spirituel/spiritualité » d'après les définitions données par Tillich (1969) et Waaijman (1993, 2004). Elles doivent être interprétées comme un questionnement à la quête de sens. Elles deviennent alors des outils de compréhension pour les adultes éducateurs en quête de solution au débat sur le religieux prenant court dans le scoutisme francophone canadien.

\section{Points de repère sur la pensée spirituelle de Baden-Powell}

La naissance du scoutisme dans le monde francophone ne s'est pas fait sans difficulté. Quand on retrace l'histoire du scoutisme, on se rappelle des tensions en ce qui concerne l'acceptation des principes proposés par le fondateur. Certains hommes d'église véhiculaient des craintes 
non fondées de peur que le scoutisme ne s'impose comme une nouvelle religion ou tout simplement par jalousie. Considérant les moeurs du début du siècle, il existait un problème de perception qui s'est relativement résorbé avec le temps quant aux intentions religieuses réelles de Baden-Powell. Nombre de catholiques ont rejeté le scoutisme tout simplement parce qu'il avait été fondé par « un "protestant," "franc-maçon," et met en oeuvre une philosophie naturaliste " (Estienne, 1994, p. 41). Cette attitude d'exclusion explique sans doute la lente implantation du scoutisme au Canada-français. Le fondateur se devait de rectifier ces problèmes de perception par un dialogue soutenu. À cet égard, il rencontre plusieurs politiciens, chefs religieux et organisateurs scouts dans différents pays pour en faire la promotion. À ses détracteurs qui lui demandaient en quoi la religion entre-t-elle dans le mouvement, il répond que son projet religieux atteste d'une bonne volonté à créer un organisme offert à la jeunesse du monde entier en affirmant que la religion « n'y entre pas du tout. Elle est déjà là. Elle est le facteur fondamental » (Baden-Powell, 1926/1982, p. 30). Le religieux est donc le fondement même du mouvement scout et a la prétention de s'adapter à une société multiculturelle. Pour Baden-Powell, il n'est pas une nouvelle religion à adhérer, mais répond au besoin chez l'enfant à penser et apprendre par lui-même en respectant son individualité:

Mais ne va pas te figurer, [...] que j'essaie de te convertir à quelque nouvelle forme de religion. Non. Je suis simplement parti de l'idée que toi qui me lis, n'a pas d'idée religieuse personnelle très profonde ou que tu t'aperçois que les athées essaient de te convaincre. Je veux simplement te suggérer que la meilleure compréhension de Dieu peut être acquise par l'étude de la nature [...]. Je sais que cela est arrivé dans bien des cas à des bûcherons, des marins, des soldats et des explorateurs qui n'avaient jamais saisi autrement la moindre notion de foi religieuse. (Baden-Powell, 1922/1946, p. 223)

D'après Baden-Powell, la mise en oeuvre de la religion est au fond une chose très simple qui réside dans l'application de deux dimensions: « Aimer et servir Dieu. Aimer et servir son prochain » (Baden-Powell, 1908/1951, p. 263). ll propose que l'enseignement de la religion soit présenté dans son aspect le plus héroîque et non pas d'une manière sinistre. Le jeune a déjà une inclination naturelle pour la religion. L'important c'est que le scout adhère à la pédagogie du mouvement qui repose sur trois principes: le devoir envers Dieu, aux autres et à soi-même (OMMS, 1992). Pour ce faire, les responsables locaux ont eux-mêmes la liberté d'en définir les amples détails: 
Il est sans doute fort difficile de donner une définition précise de la formation religieuse dans notre mouvement, car il y coexiste des confessions fort différentes. C'est la raison pour laquelle les détails de l'expression du devoir envers Dieu doivent être laissés dans une large mesure entre les mains des responsables locaux du mouvement. Mais nous insistons sur un point: le garçon doit observer et mettre en pratique la religion qu'il professe, quelle qu'elle soit. (Baden-Powell, 1944/1982, p. 128)

Baden-Powell témoigne ainsi d'un profond respect face aux différentes confessionnalités qui se côtoyaient dans l'Angleterre protestante au début du siècle. Il assure aux scouts l'appartenance à une confessionnalité en acceptant, en même temps, le phénomène du pluralisme religieux. Sur ce point, Baden-Powell devance son époque. Concernant les observances religieuses, il résume la ligne de conduite des scouts par l'application de principes qui permet de poursuivre l'idéal de paix internationale et de fraternité humaine. Ainsi, chaque scout est convoqué à s'identifier à une confession religieuse et à suivre les rites cultuels. Si une unité est composée de scouts appartenant à plusieurs confessions différentes, il n'est pas permis d'organiser de service en commun et la prière pratiquée est la plus simple possible tout en laissant la liberté à chacun d'y participer ou non. Là où les règles d'une confession religieuse qui ne permettent pas au scout de participer au culte qui n'est pas le sien, les adultes éducateurs tâcheront alors que ces règles soient observées (BadenPowell, 1908/1951). Ce qui importe c'est que le jeune pratique sa religion d'après son appartenance dans le respect de ce que sont les autres. Ces quelques principes sont toujours mis en application dans le scoutisme francophone canadien.

Profondément chrétien, Baden-Powell ne partage pas les perspectives légalistes d'une dénomination chrétienne particulière. Son approche s'appuie sur la quête de Dieu ou selon le sens de l'inscription des humains dans le monde. Il tient pour acquis et respecte les différentes croyances religieuses ainsi que les façons distinctes d'appliquer les différentes pratiques rituelles. Selon lui, la pédagogie scoute doit favoriser une dynamique religieuse qui s'inscrit au coeur même de la vie quotidienne. Ainsi, Baden-Powell affirme que « si l'on traitait davantage la religion comme faisant partie de la vie quotidienne, elle n'y perdrait rien de sa dignité et elle y gagnerait de l'emprise sur les hommes » (Baden-Powell, $1908 / 1951$, p. 326). Les activités scoutes sont alors d'autres lieux où se vivent le religieux. Dans cette perspective, Baden-Powell crée une autre dimension du religieux en essayant d'être toujours en lien avec les religions institutionnalisées. 
La quête spirituelle de Baden-Powell est donc multiconfessionnelle. Cette notion apparaît primordiale dans les rencontres mondiales des chefs scouts. Certains principes directeurs guident la pédagogie du développement spirituel. Ainsi, le mouvement recherche-t-il la fraternité et la camaraderie universelle entre tous les membres de toutes nations, de toutes classes et de toutes religions. Le mouvement se veut national en vue de former des citoyens du monde utiles et sains. Il ne cherche pas à affaiblir, mais plutôt à reconnaître les croyances religieuses respectives de ses membres. Finalement, on demande à chaque scout de pratiquer avec fidélité et sincérité sa religion et de s'interdire toute propagande confessionnelle lors des réunions où se trouvent d'autres scouts issus d'une autre confessionnalité (Bureau mondial du scoutisme [BMS], 1985). Les liens de camaraderie entre les nations deviennent alors réalisables. Ce faisant, il est possible de participer à la fraternité universelle que propose le fondateur tout en respectant sa propre identité religieuse.

Pour cet humaniste anglais, la religion se situe au plan de l'intuition. Elle ne doit pas être imposée ni constituer une science réservée à des gens instruits ni considérée comme un vêtement du dimanche, mais plutôt « une dimension fondamentale de la personnalité du garçon, un développement de l'âme, et non un revêtement qui peut se détacher. C'est une question de personnalité, de conviction intérieure, et non d'instruction du garçon » (Baden-Powell, 1944/1982, p. 129). La religion n'est pas uniquement réservée aux spécialistes ou aux faibles d'esprit. Elle surgit de l'intérieur en favorisant le développement de la personnalité chez le jeune. Le scoutisme promulgue une pédagogie de l'accompagnement plutôt qu'une pédagogie de l'enseignement.

Pour Baden-Powell, Dieu ne se limite pas à des questions de dogmes, de foi ou d'appellation qui peuvent empêcher la libre circulation du religieux. Au contraire, l'image de Dieu revêt un visage plus humain « c'est "la parcelle divine" qui est en chacun de nous, c'est notre âme " (BadenPowell, 1922/1946, p. 222) et qui se révèle dans la charité et l'amour du prochain. Plus le scout est disposé à partagér cet amour évangélique, plus son âme se développe et croît en sagesse en union intime avec Dieu et l'éternité.

La vision spirituelle de Baden-Powell propose une rupture d'avec le fondamentalisme religieux de son époque. Il va même plus loin par l'intuition fondamentale des liens pouvant exister entre l'expérience religieuse et le jeu. Ses expériences de jeunesse intégrées par le goût de l'aventure sont les pierres d'assise de sa spiritualité du jeu. C'est d'ailleurs dans le terrain de jeux que se construira son identité qui lui per- 
mettra de trouver sa vocation. Ses souvenirs d'enfance déterminent sa destinée en ce qui à trait à l'oeuvre qu'il édifiera par le scoutisme:

Ami lecteur, j'ignore ce qu'ont pu être tes expériences, mais quant à moi, le nombre des choses pratiques de la vie que j'ai apprises à l'école est minime comparé à celui que j'ai appris par moi-même sur le terrain de jeux. C'est là que mes angles ont été rabotés, que j'ai trouvé ma place et que mon caractère a commencé à être formé. Et, quoique les jeux euxmêmes n'aient pas d'utilité pour l'âge moyen de la vie, ils m'ont été utiles à ce stade de préparation et leurs leçons m'ont été durables. C'est dans les bois qui environnaient l'école et le terrain de jeux que j'ai appris la plupart des choses que je sais. Ce que j'ai appris dans le taillis était à la fois une préparation et une activité qui m'ont été utiles jusqu'à aujourd'hui. Cette vie du fourré me plaisait et m'instruisait tout à la fois, et par cela même elle resta gravée en moi. Cette vie dans la nature favorisa non seulement le développement du corps, de la santé et de l'esprit, mais elle m'aida comme jeune garçon, à trouver mon âme. (Baden-Powell, 1927/ 1946, pp. 22-23)

L'expérience de Baden-Powell est ici significative pour le développement de sa pensée sur le jeu lequel Huizinga (1951, p. 329) soutient que « le mouvement s'intitule jeu lui-même. " Pour Baden-Powell,

le jeu est le premier éducateur. Cela est vrai des animaux sauvages aussi bien que des hommes. [...] Ainsi en est-il de nos Louveteaux à nous. Nous leur enseignons en jouant de petites choses qui les rendront capables de faire sérieusement de grandes choses quand le moment sera venu. (Baden-Powell, 1916/1961, p. 215)

Il a l'ultime conviction que le jeu/loisir possède une dimension fondamentalement religieuse et spirituelle que l'on retrouve également dans la littérature scientifique. Celui-ci est une « attitude spirituelle et mentale », une « attitude de l'esprit » et une « disposition de l'âme » (Pieper, p. 40). Le jeu/loisir est donc une activité contemplative qui affirme l'être et la création de la culture. Il « implique (en premier lieu) une attitude de non-activité, de calme intérieur, de silence; il signifie ne pas être "occupé," mais laisser les choses arriver d'elles-mêmes " (Pieper, 1952, p. 41). D'une manière analogue, le jeu/loisir est un "état d'esprit » (Neulinger, 1974, p. xii) et rejoint donc la compréhension moderne du religieux puisqu'il est « une façon d'être en paix avec soi-même et avec ce qu'on est en train de faire » (Neulinger, p. xvii). Cela suppose l'engagement de l'être dans ses profondeurs et la responsabilisation sans pression extérieure. Le jeu/loisir permet alors d'exprimer son plein potentiel et ses talents pour sa propre fin et est « relié à la quête de trou- 
ver Dieu ", « créer/découvrir le sens de la vie » et " relié avec nousmêmes/autres » (Karlis, Grafanaki, \& Abbas, 2002, p. 212). Dans cette perspective, la mise sur pied des projets scouts par les jeunes et les adultes éducateurs selon les principes fondamentaux font d'eux des chercheurs de Dieu dans une dynamique ludique. Ainsi, Baden-Powell développe une image de Dieu qui ne s'intéresse pas seulement à notre côté religieux, mais qui peut se rencontrer au coeur des activités humaines. Il avance que l'une des clefs du bonheur, c'est de considérer la vie comme un grand jeu et la vie d'équipe, un grand jeu qui selon les adultes éducateurs représente une méthode pour développer le sens des responsabilités chez les jeunes. Le scout est donc appelé à prendre sa place dans le jeu imaginé et organisé pour faire de lui un humain à part entière dans la communauté. En ce sens, sa pensée sur le jeu véhicule une forte connotation spirituelle:

Notre travail devient facile quand nous le considérons comme un jeu où nous sommes les joueurs d'une équipe, chacun jouant à sa place, tous jouant ensemble pour le bien du camp, et quand l'esprit d'équipe s'empare de nous, nous découvrons que ce n'est pas seulement un jeu, mais un grand jeu. [...] Nous nous y mettons gaîment avec le sourire et quand un travail est fait avec le sourire, les difficultés s'évanouissent. Le travail devient alors un plaisir, un jeu. [...] Ainsi jouez, et jouez le jeu! Jouez à votre place, non pour vous-même, mais pour votre équipe, pour votre pays, pour vos semblables. (Baden-Powell, 1927/1946, pp. 57-58, 61)

Baden-Powell est celui qui a aimé atteindre des sommets tant professionnels que personnels. Il aimait grimper des montagnes lorsqu'il affirme que l'alpinisme l'attire « non seulement pour le plaisir de l'escalade, mais parce qu'il comporte quelque chose de spirituel et d'exaltant, aussi salutaire à l'âme que l'exercice est salutaire au corps » (BadenPowell, 1933/1946, p. 91). Baden-Powell est donc un grand fervent de la nature. Pour lui, le jeune a plus de chance d'y rencontrer Dieu que dans le cadre étouffant des sermons et l'atmosphère malpropre des taudis: "Le but de l'étude de la nature est de donner à chacun le sens de la beauté de la création et de lui faire réaliser l'existence de son Créateur » (Baden-Powell, 1918/1923, p. 125). Pour éviter l'athéisme, il suggère un va et vient entre la Bible et la nature (Baden-Powell, 1922/1946, p. 198). Ainsi, le cadre de la nature devient-il un lieu de prédilection pour vivre une dynamique ludique avec Dieu et comporte une dimension spirituelle non négligeable (Heintzman, 2000; Deschênes, 2002). Dans le lieu cosmique de la nature, les scouts vivent des expériences de Dieu tel que l'entend Baden-Powell: 
Ton sac à dos et ton bâton en main ou « ta petite monture d'acier entre les jambes emportant avec toi ta petite maison de toile, ta couverture et ta gamelle, et...la liberté. Dans la nature, au milieu des œuvres de Dieu surtout « en camp volant », c'est-à-dire en courses à travers la campagne, t'abreuvant des merveilles du ciel, de la terre et de la mer, voyant les couleurs des bois et des champs, respirant le parfum des fleurs et du foin, entendant la musique des ruisseaux, le chant des oiseaux et le murmure du vent, apprenant à connaître les animaux et leurs moeurs jusqu'à ce que tu sentes leur camarade et une partie du plan grandiose de la nature. (Baden-Powell, 1922/1946, pp. 210-211)

Cette pensée de Baden-Powell d'un va et vient entre la Bible et la nature constitue une idée maîtresse reprise dans la pensée humaine universelle. Les écrits mystiques, théologiques, philosophiques, littéraires, scientifiques ou provenant d'autres horizons spirituels les plus divers proposent en eux-mêmes toutes une phénoménologie religieuse où l'on découvre la sagesse mystérieuse du Créateur qui se révèle dans le monde. Dans sa théorie de la révélation transcendantale, $\operatorname{Rahner}(1983$, p. 231) développe la notion théologique moderne de l'" autocommunication de Dieu » comme créateur et sauveur dans le Christ Jésus qui accomplit l'autotranscendance du monde en lui et vers lui. L' « autocommunication de Dieu » est alors un mystère intime qui vient habiter la matière dans le monde. Dans la révélation de Dieu dans l'histoire de la création et du salut, Dieu est transcendant au sein de son immanence: « Dieu le créateur du ciel et de la terre est présent par son Esprit cosmique dans chacune de ses créatures et dans la communauté créée » (Moltmann, 1988, p. 28). Cette communicabilité de Dieu dans la création est une notion de perception avec les sens dont l'humain possède à travers l'expérience qui s'inscrit dans une "esthétique de la révélation » (Waldenfels, 1990, p. 255). Dans cette perspective, relisons quelques descriptions comme l'atteste la forte correspondance de quelques citations du « livre de la Bible » et les réflexions historiques de penseurs sur le « livre de la nature » qui évoquent tout le potentiel d'apprendre de la création de Dieu.

Ce qui est à comprendre dans ces descriptions c'est « la chose du texte, à savoir la sorte de monde que l'oeuvre déploie en quelque sorte en avant du texte " (Ricoeur, 1986, p. 168). C'est donc un monde de loisir qui se déploie devant nous, car le monde dont parlent ces auteurs est différent du monde ordinaire. Dans ces discours sur le « livre de la nature ", on associe ces expériences à un sentiment de bien-être, de gratuité, d'intériorité, de dépassement ou de transformation. Ils sont d'ordre extatique et contemplatif c'est-à-dire un état où les gens se 


\section{Tableau 1}

\section{Le livre de la Bible}

« Dieu dit: "Faisons l'homme à notre image, selon notre ressemblance et qu'il soumette les poissons de la mer, les oiseaux du ciel, les bestiaux, toute la terre et toutes les petites bêtes qui remuent sur la terre!" Dieu créa l'homme à son image, à l'image de Dieu il le créa: mâle et femelle il les créa. Dieu les bénit et Dieu leur dit: "soyez féconds et prolifiques, remplissez la terre et dominez-la. Soumettez les poissons de la mer, les oiseaux du ciel et toute bête qui remue sur la terre!" " (Gn 1, 26-28).

«Les cieux racontent la gloire de Dieu, le firmament proclame l'oeuvre de ses mains ") (Ps 19, 1).

« Mais interroge denc les bestiaux, ils $t$ 'instruiront, les oiseaux du ciel, ils t'enseigneront. Cause avec la terre, elle t'instruira, et les poissons de la mer te le raconteront. Car lequel ignore, parmi eux tous, que “c'est la main du Seigneur qui fit cela." Lui qui tient en son pouvoir l'âme de tout vivant et le souffle de toute chair d'homme » (Job 12, 7-10).

" J'entends mon chéri! Le voici: il vient! Sautant par-dessus les monts, bondissant pardessus les collines, mon chéri est comparable à une gazelle ou à un faon de biche. Le voici: il s'arrête derrière notre mur; il regarde par la fenêtre; il épie par le treillis. Mon chéri chante et me dit: "Debout, toi, ma compagne, ma belle, et viens-t'en. Car voici que l'hiver passe; la pluie cesse, elle s'en va. On voit les fleurs dans le pays; la saison de la chanson arrive; et on entend dans notre pays la voix de la tourterelle " (Ct 2, 8-12).

«Quand Il affermit les cieux, moi, j'étais là, quand Il grava un cercle face à l'abîme, quand Il condensa les masses nuageuses en haut et quand les sources de l'abîme montraient leur violence; quand Il assigna son décret à la mer et les eaux n'y contreviennent pas, quand II traça les fondements de la terre. Je fus maître d'oeuvre à son côté, objet de ses délices chaque jour, jouant à sa présence en tout temps, jouant dans son univers terrestre; et je trouve mes délices parmi les hommes » $(\operatorname{Pr~8,~30-31).~}$

\section{Réflexions sur le livre de la nature}

«Dieu est le Créateur de l'univers » (St Irénée de Lyon, Against Heresies).

"La nature est notre meilleure enseignante » (St Jean Chrysostome, On the Statufes)

«Certaines personnes lisent des livres en vue de connaître Dieu, mais il y a un grand livre: l'avènement des choses créées " (St Augustin, De Civit Dei)

« Vous trouverez quelque chose de plus grand dans les bois que dans les livres. Les arbres et les cailloux vous enseigneront autant que vous n'avez jamais appris des maîtres ) (St Bernard de Clairvaux, The Letters of Bernard)

«Chaque feuille de l'arbre devient une page de l'écriture sacrée depuis que l'âme a appris à les lire " (Cheik Muslih-uddin Sa'di Shirazi, The Gulistan).

« Toute créature est un livre à propos de Dieu » (Maitre Eckhart, Sermons)

« Dieu écrit dans l'Évangile non seulement dans la Bible, mais également dans les arbres, les fleurs, les nuages et les étoiles 》 (Martin Luther, cité in The Harper Religious and Inspirational Quotation Companion).

«Le livre de la nature est une précieuse et grande pièce de tapisserie enroulée qu'on ne peut voir d'un seul coup mais qui doit être patienté pour la découverte de son contenu et de sa beauté lorsqu'elle se déplie davantage peu à peu graduellement et symétriquement ") (Robert Boyle, The Christian Virtuoso).

« Le pays est à la fois le jardin du philosophe et sa librairie dans laquelle il lit et contemple la puissance, la sagesse et la bonté de Dieu » (William Penn, Some Fruits of Solitude).

« Le livre de la création est en accord avec le livre écrit et la révélation sacrée de tous les prophètes de Dieu " (Abdu'l-Baha, Makatib). 


\section{Tableau 1 (continued)}

\section{Le livre de la Bible}

« Car la grandeur et la beauté des créatures conduisent par analogie à contempler leur Créateur » $(\mathrm{Sg} \mathrm{13,5})$.

« Et du vêtement, pourquoi vous inquiéter? Observez les lis des champs, comme ils croissent: ils ne peinent ni ne filent, et je vous dis, Salomon lui-même, dans toute sa gloire, n'a jamais été vêtu comme l'un d'eux! " (Mt 6, 29-29).

Source: Traduction Oecuménique de la Bible (1988).

\section{Réflexions sur le livre de la nature}

« La joie qui vient à la lecture de la création de Dieu " (George Carver, George Washington Carver In His Own Words).

« La nature dans son entier et dans tous ses éléments expriment quelques chose qui peut être vu comme une auto-communication indirecte de Dieu à ceux qui sont prêts à le recevoir » (Martin Buber, At the Turning).

Source: Opening the Book of Nature (2006). (Citations en traduction libre).

retrouvent, à divers niveaux, comme entraînés hors de soi. Ainsi, il importe de souligner que ces discours ouvrent à une expérience de profondeur et de transcendance.

Cette analogie sémantique entre le « livre de la Bible » et le « livre de la nature » que Baden-Powell avait expérimenté donne à penser à une vision du monde sous la sagesse de Dieu. Plusieurs théoriciens du jeu viennent alimenter la pensée spirituelle de Baden-Powell dû au fait que le scoutisme propose un royaume de l'enfance comme manière d'être où le jeu est « un processus à travers lequel un sujet se réalise lui-même dans une dialectique de décision et de surprise" (Lites, 1992, p. 39). Accéder au jeu de la création, c'est sortir de la vie fonctionnelle et s'imposer des surprises; c'est se rendre compte à l'intérieur que l'on peut être autrement dans un monde de gratuité et de grâce. Dans cette perspective, le jeu de la création peut donner lieu à l'imagination, à l'action et à l'authenticité. Ainsi, " parler du jeu, c'est parler de la nature de l'humain » (Neale, 1969, p. 21). C'est ce que le Christ Jésus voulait dire dans cette affirmation évangélique: "Laissez les enfants venir à moi; ne les empêchez pas, car le Royaume de Dieu est à ceux qui sont comme eux » (Lc $18,16)$. L'aspect esthétique du jeu fait participer l'enfant à l'aventure de la création. Le jeu est un symbole cosmique du monde (Moltmann, 1988) puisqu'il est l'illustration qui permet de comprendre l'existence humaine dans le temps du monde. C'est en jouant que l'on se libère de la pesanteur de l'existence et l'on s'amuse ainsi à voir les choses sous un autre angle. Dans l'acte de « jouer, c'est se soumettre à une sorte de magie, se représenter soi-même l'absolument autre, par avance acqué- 
rir l'avenir, démentir le gênant monde des faits " (Rahner, 1967, p. 65). Il n'y a ni maître ni valet dans le jeu de la création. On est comme des enfants pénétrés de la « confiance originaire » (Küng, 1981, p. 524) qui découvre que « la création est un jeu de Dieu, un jeu de sa sagesse infinie et insondable. Elle est elle-même l'espace où se déploie en se jouant la magnificence de Dieu i) (Moltmann, 1977, p. 40). La création est donc une action libre, volontaire et gratuite du bon plaisir de Dieu; une action de Sa volonté amoureuse, non un accident de parcours ni un hasard.

Cette perception du monde comme création éveille la joie de vivre et correspond au dernier message de Baden-Powell adressé à la communauté scoute: «Je crois que Dieu nous a placés dans ce monde merveilleux pour y être heureux et jouir de la vie. La meilleure manière d'atteindre le bonheur est de le donner aux autres » (Baden-Powell, $1908 / 1951$, p. 332). Cette quête de liberté qui suscite la joie de vivre approfondie le jeu de la création. En ce sens, Lee (1964) suggère quatre attitudes à adopter pour un jeu/loisir créateur: (a) d'accepter le temps que Dieu accorde aux humains, (b) de vivre selon la qualité des événements, (c) d'avoir la volonté à s'engager dans l'action, (d) d'être ouvert à la joie de vivre dans l'amour créatif. Ces attitudes sont nécessaires à la réalisation de soi. Le jeu/loisir créateur devient alors l'opportunité et la capacité de devenir selon la volonté de Dieu (Spence, 1973). Ce processus de devenir humain par le jeu/loisir est « une activité qui construit la communauté et développe l'intégralitẻ de soi-même dans la grâce et la capacité d'aimer » (Kelly, 1987, p. 225). Dans le jeu/loisir, l'action existentielle peut créer une dimension de soi-même ou une communauté qui donne un sens à la vie. Le jeu/loisir est la liberté pour être et pour devenir, pour soi-même et pour la société. Cette réalité a été profitable pour plusieurs hommes et femmes d'action dans l'histoire du scoutisme. Cela leur a permis d'atteindre le processus d'humanisation par la créativité. Elle est un lieu fondamental d'ouverture, d'autonomie et de restitution de l'humain. Le scout découvre alors sa réalité sociale et existentielle afin de faire des découvertes sur son identité et construire sa communauté.

Le développement de la spiritualité du jeu ne va pas sans considérer la qualité du sens de l'humour que porte la pédagogie de BadenPowell. Selon lui, l'humour atténue la tragédie humaine. Elle permet de surmonter les limites de la finitude sans trop se prendre au sérieux. Berger (1972, p. 112) avance même que l'humour devient un « indice de transcendance » qui prend racine dans la foi et favorise le dépassement 
du nihilisme ambiant. Baden-Powell enseigne à maints égards de cultiver ce que Cox $(1971$, p. 185) nomme « l'espérance comique » qui allège du désenchantement et de l'absurde. L'humour est un moyen de se détacher des problèmes de l'existence et de la dureté de la vie. Cette qualité humaine demeure un don que Baden-Powell cherche à transmettre aux membres de son mouvement:

Riez tant que vous pouvez: cela fait du bien; toutes les fois que vous pouvez rire de bon coeur, allez-y. Et faites aussi rire les autres autant que possible, car cela leur fera du bien. Si vous êtes dans la peine ou dans l'embarras, efforcez-vous de sourire; si vous vous en souvenez et que vous en soyez capable, vous verrez que cela fait vraiment une différence. (BadenPowell, 1908/1951, p. 232)

Baden-Powell estime que l'application du religieux dans le mouvement s'actualise dans l'acte du service, modus vivendi du scoutisme. Ainsi, ce qui reflète le plus l'image du scoutisme aujourd'hui et donne toute sa crédibilité, c'est la « bonne action » qui est bien le corollaire du service aux pauvres prôné par le Christ Jésus. En fait, la « bonne action » est un geste important au coeur du scoutisme, le scout y apprend à servir. Celui-ci s'engage à faire une " bonne action » à chaque jour, peu importe son importance, jusqu'à ce que cela devienne une habitude. « Il peut ne s'agir que d'aider une vieille femme à traverser la rue ou de dire du bien de quelqu'un qu'on dénigre. Ce qui compte, c'est de faire quelque chose » (Baden-Powell, 1901, p. 17). L'observance quotidienne de la « bonne action » devient alors une manière d'être dans le monde. Baden-Powell dit à ce sujet:

[...] si tu te mets au service des autres tous les jours dans les petites choses comme dans les grandes, tu t'apercevras que l'étincelle d'Amour se développe en toi à tel point qu'elle te permet de franchir, le coeur gai, toutes les petites difficultés et les petits ennuis de la vie ; tu te sens au-dessus d'eux, tu es plein de bonne volonté envers les hommes, et la conscience, la voix intérieure, te dit: « C'est bien! » (Baden-Powell, 1922/1946, p. 222)

L'instauration de la loi scoute actualise « le jeu du scoutisme » (Baden-Powell, 1936/1937, p. 187). À l'origine, Baden-Powell s'était inspiré du code d'honneur des chevaliers pour formuler la loi scoute qui a subi certaines transformations au cours du temps en s'adaptant aux différents groupes d'âge où se pratique le scoutisme. La formulation de la loi scoute prend diverses formes selon la culture du pays. L'Association des Scouts du Canada formule la loi scoute, en ses différents articles, d'après chaque groupe d'âge qu'elle dessert. 


\section{BRANCHES}

Hirondelles (7-8 ans)

Castors ( $7-8$ ans)

Exploratrices (9-11 ans)

Louveteaux (9-11 ans)

Intrépides (11-14 ans)

Éclaireurs (11-14 ans)

Pionniers (14-17 ans)

Pionnières (14-17 ans)

Scouts-Aînés (17-21 ans)

\section{LOI}

L'hirondelle est amicale et enjouée.

Le castor joue avec et comme les autres. Je suis propre. Je suis vraie. Je suis active. Je suis joyeuse. Je suis l'amie de l'autre. Le louveteau fait de son mieux.

Le louveteau fait plaisir à quelqu'un tous les jours.

Je répands la joie. Je partage avec les autres. Je protège l'environnement. Je fais équipe. Je mérite et fais confiance. Je respecte les différences. Je défends ce qui est juste. Je vis ma foi. J'acquiers la maîtrise de moimême. Je poursuis ce que j'entreprends.

Le scout mérite et fait confiance.

Le scout combat pour la justice.

Le scout partage avec tous.

Le scout est frère de tous.

Le scout protège la vie.

Le scout fait équipe.

Le scout fait de son mieux.

Le scout répand la joie.

Le scout respecte le travail.

Le scout est maître de lui-même (ASC, 2000 , p. 10).

La loi scoute se traduit par un code moral positif, c'est-à-dire qu'il n'y a pas d'interdit. Il n'y a pas d'autorité extérieure pour la faire respecter. Seule la responsabilité personnelle est convoquée áu « jeu du scoutisme ». À partir de là, la régulation organisationnelle du scoutisme n'obéit pas à une dynamique hétérorégulatoire, mais adopte surtout une approche autorégulatoire qui interpelle la liberté personnelle (Giroux, 1997). Celle-ci doit cheminer dans le dialogue moral dans la communauté en respectant ce que sont les autres dans leurs différences, leurs difficultés et leurs forces.

\section{Discussions et conclusion}

Suite à l'explication des points de repère sur la pensée spirituelle de Baden-Powell, il est permis d'affirmer que, pour l'époque, ses idées sur la religion appelle à un changement de paradigme. Il propose à la jeunesse mondiale de grands principes afin d'être religieux autrement. Plu- 
tôt que par l'apprentissage de dogmes, la religion, loin d'être contrôlé par la morale des églises, peut être intégrée à l'expérience de la vie, du jeu, de la nature et du service rendu. Certes, Baden-Powell respecte les différentes confessions religieuses et les pratiques rituelles, mais il a aussi l'intuition qu'il y a d'autres lieux du religieux. Le scoutisme est multiconfessionnel et s'adapte au phénomène du multiculturalisme lorsque l'on applique les principes de base pour atteindre la fraternité universelle tant désirée par le fondateur du mouvement scout.

Le scoutisme francophone canadien pour sa part est en quête d'identité cherchant l'unité dans la diversité. Cela ne va pas sans les conflits de valeur propres aux changements de mentalité qui en génèrent dans un contexte multiculturel en constante évolution. Les concepts modernes " religion/religieux » et «spirituel/spiritualité » sont parfois mal compris par certains adultes éducateurs qui veulent le contrôle du religieux et empêchent ainsi sa libre circulation. Il s'agit de ne pas oublier l'intention première du fondateur lorsqu'il affirme que le but du mouvement: « est de contribuer, dans la mesure du possible, à réaliser le Royaume de Dieu sur la terre, en développant parmi les jeunes l'esprit et la pratique de la bonne volonté et de la coopération à l'égard des autres dans la vie quotidienne » (Baden-Powell, 1926/1982, pp. 139-140). À partir de là, il est possible de dépasser la double tension qui exist dans le débat du scoutisme francophone canadien. Entre ceux qui veulent complètement laîciser le mouvement et les autres qui appellent à garder l'identité chrétienne catholique, la nécessité de retourner aux textes fondateurs demeure une condition importante à la compréhension de la pensée spirituelle de Baden-Powell pour une ouverture acceptable aux différentes confessions religieuses et de l'esprit laïc qui se côtoient dans le scoutisme. De plus, le dialogue moral dans le débat est nécessaire à la croissance spirituelle des scouts qui adhèrent à une pensée européenne dans le contexte multiculturel nord-américain. Prendre conscience de cette réalité permet d'éviter les possibles dérapages surtout lorsqu'il devient impératif que les délibérations et les prises de décisions soient dictées par des valeurs de tolérance et de charité. Une éthique de la neutralité de la part des adultes éducateurs est décisive dans leur intervention auprès des scouts provenant de confessions religieuses différentes si l'on veut garder intactes les intentions premières du fondateur. Chose certaine, il n'y a pas de scoutisme sans développement spirituel. Que les adultes éducateurs " apprennent plutôt à se perdre et ils se retrouveront dans ce qu'ils ont à devenir, en aidant le monde à répondre à sa fin ") (Valadier, 1987, p. 238). En recherchant le dialogue moral, les adultes éducateurs auront 
alors la possibilité de retrouver la source de leur identité en répondant aux questions spécifiques du religieux dans le scoutisme tout en respectant les principes fondamentaux émis par le fondateur.

Baden-Powell a l'intuition qu'il faut considérer la vie comme un grand jeu qui s'inscrit dans la découverte de Dieu et devient alors le dénominateur commun de la spiritualité dans le scoutisme. Fondamentalement, les activités scoutes s'inscrivent dans le jeu/loisir où Dieu joue avec les scouts dans la racine de leur identité véritable. Les scouts découvrent donc par eux-mêmes qu'ils participent à une spiritualité porteuse de sens et qui permet la découverte de leur propre royaume. Cela se réalise lorsque les scouts apprennent à la suggestion de Baden-Powell de faire un va et vient entre la Bible et la nature. Appuyé par une théologie de la création et des théoriciens du jeu/loisir, le cadre naturel fait des scouts des chercheurs de la sagesse mystérieuse de Dieu dans une dynamique ludique où la révélation transcendantale a une valeur esthétique. Il est alors possible d'apprendre du jeu de la création qui donne lieu à l'imagination, à l'action et à l'authenticité. La spiritualité du jeu est une activité qui affirme l'être et la création de la culture. Il fait participer les scouts à l'aventure de la création dans leur processus de devenir et de construction de leur communauté dans une quête de liberté qui suscite la joie de vivre.

La spiritualité du jeu dans le scoutisme apparait comme une authentique renaissance culturelle, permettant aux scouts d'enrichir leurs connaissances et d'aller à la rencontre d'autres cultures et religions. Le scoutisme devient un lieu de convivialité, d'amitié, de participation engagée, de solidarité et d'intégration sociale. Cette approche spirituelle se révèle alors comme une nouvelle manière de vivre dans le monde, une relation étroite entre la grâce, le don et la gratuité. La spiritualité du jeu tel que proposée par Baden-Powell nécessite de vivre, de prendre son temps et d'être-soi-même. Elle est également un « état d'esprit » procurant un sentiment de mieux-être et veut répondre à la question du « comment vivre $»$ ? C'est une quête qui relève de la créativité, de la responsabilité et de la coopération.

Baden-Powell ne doit pas être considéré comme un théoricien. Son approche est pragmatique et permet la résolution de problèmes pratiques. Les points de repères de sa pensée spirituelle ouvre à la découverte d'une spiritualité du jeu et l'application des principes fondamentaux est un point de départ face aux difficultés que rencontrent les adultes éducateurs devant le pluralisme religieux. Il faut savoir que sa spiritualité du jeu est difficilement mesurable scientifiquement par l'approche 
quantitative. Il y aurait toutefois tout un champ d'exploration à réaliser pour la recherche qualitative. Cela permettrait d'approfondir et d'appliquer sa pensée toujours actuelle dans le monde d'aujourd'hui. Il existe donc un intérêt notable pour le monde de la connaissance et de la pratique qui ont la possibilité de mieux comprendre le phénomène de la libre circulation du religieux et de ses aboutissants dans la société multiculturelle canadienne à partir d'une organisation de jeunesse qu'est le mouvement scout.

\section{Références}

Association des scouts du Canada. (1999). Azimuts. Montréal, QC: ASC. Association des scouts du Canada. (2000). Programme des jeunes 1: ANI 1026. Montréal, QC: ASC.

Association des scouts du Canada. (2007). Rapport annuel. Montréal, ASC.

Baden-Powell, R. (1899). Aids to scouting for NCO's and men. London: Gale \& Palden.

Baden-Powell, R. (1901). Extrait d'une lettre écrite du Transvaal. Facets. London.

Baden-Powell, R. (1908/1951). Scouting for boys (Éclaireurs). Neuchatel, Suisse: Delachaux \& Niestlé S.A.

Baden-Powell, R. (1918/1923). Girl guiding (Le livre des éclaireuses). Neuchatel, Suisse: Delachaux \& Niestlé S.A.

Baden-Powell, R. (1916/1961). The wolf cub's handbook (Le livre des louveteaux). Neuchatel, Suisse: Delachaux \& Niestlé S.A.

Baden-Powell, R. (1922/1946). Rovering to success (La route du succès). Neuchatel, Suisse: Delachaux \& Niestlẻ S.A.

Baden-Powell, R. (1926/1982). Discours à la Conférence des Commissaires scouts et guides. Dans M. Sica (Trad. \& Dir.), Jouer le jeu (pp. 30, 139-140). Paris: Scouts de France.

Baden-Powell, R. (1927/1946). Life's snags and how to meet them (Franchis l'obstacle). Neuchatel, Suisse: Delachaux \& Niestlé S.A.

Baden-Powell, R. (1933/1946). Lessons from the varsity of life (À l'école de la vie). Neuchatel, Suisse: Delachaux \& Niestlé S.A.

Baden-Powell, R. (1936/1937). Adventuring to manhood (Pour devenir un homme). Neuchatel, Suisse: Delachaux \& Niestlé S.A.

Baden-Powell, R. (1944/1982). Aids to Scoutmanship. Dans M. Sica (Trad. \& Dir.), Jouer le jeu (pp. 128-129). Paris: Scouts de France.

Béland, J. P. (1988). Quand je dis ma foi. Paris/Montréal: Médiapaul/Paulines.

Berger, P. (1971). La religion dans la conscience moderne. Paris: Centurion.

Berger, P. (1972). La rumeur de Dieu, signes actuels du surnaturel. Paris: Centurion.

Bernard, C.-A. (1986). Traité de théologie spirituelle. Paris: Cerf. 
Bureau mondial du scoutisme (1985). Principes du scoutisme, article 14/24. Résolutions de la conférence mondiale du scoutisme 1922-1985. Genève, Suisse.

Cox, H. (1971). La fête des fous: Essai théologique sur les notions de fête et de fantaisie. Paris: Seuil.

Deschênes, G. (2002). Pour une spiritualitẻ du loisir. Loisir et société/Leisure and Society, 25(1), 41-69.

Dumont, F. (1987). L'institution de la théologie: Essai sur la situation du théologien. Montréal, QC: Fides.

Estienne, B. (1994). De l'espérance scoute à l'espérance chrétienne. Dans G. Cholvy \& M.-T. Cheroute (Dir.), Le Scoutisme (pp. 37-54). Paris: Cerf.

Fink, E. (1966). Le jeu comme symbole du monde. Paris: Minuit.

Giroux, G (1997). La demande sociale de l'éthique. Dans G Giroux (Dir.), La pratique sociale de l'ethique (pp. 27-47). Montréal, QC: Bellarmin.

Gravelle, F. \& Deschênes, G (2004). Spiritual development through play in the outdoors: A look at the scout movement. Pathways: The Ontario Journal of Outdoor Education, 16(4), 22-25.

Heintzman, P. (2000). Leisure and spiritual well-being relationships: A qualitative study. Loisir et société/Leisure and Society, 23(1), 41-69.

Huizinga, J. (1951). Homo ludens: Essai sur la fonction sociale du jeu. Paris: Gallimard.

Karlis, G., Grafanaki, S., \& Abbas, J. (2002). Leisure and spirituality: A theoritical model. Loisir et société/Society and Leisure, 25(1), 205-214.

Kelly, J.R. (1987). Freedom to be: A new sociology of leisure. New York: Macmillan.

Küng, H. (1981). Dieu existe-t-il: Réponse à la question de Dieu dans les temps modernes. Paris: Cerf.

Lee, R. (1964). Religion and leisure in America: A study in four dimensions. New York: Abingdon Press.

Lites, W.W. (1992). Play as a metaphor for the process of perceiving God. Thèse de doctorat, Louisville, KY : The Southern Baptist Theological Seminary.

Maxence, P. (2003). Baden-Powell. Paris: Perrin.

Moltmann, J. (1977). Le Seigneur de la danse. Paris: Cerf-Mame.

Moltmann, J. (1988). Dieu dans la création. Paris: Cerf.

Neale, R.E. (1969). In praise of play: Toward a psychology of religion. New York: Harper \& Row.

Neulinger, J. (1974). The psychology of leisure. Springfield, IL: Charles C. Thomas.

Organisation Mondiale du Mouvement Scout (1992). Principes fondamentaux. Genève, Suisse: Bureau mondial du Scoutisme. 
Opening the Book of Nature (2006). Nature as a great book. Informations tirées le 27 Déc., 2007, de http://www.bookofnature.org/library/ngb .html.

Picoche, J. (1991). Dictionnaire étymologique du français. Paris: Le Robert. Pieper, J. (1952). Leisure: The basis of culture. New York: Pantheon Books. Rahner, H. (1967). Man at play. New-York: Herder \& Herder, 1967.

Rahner, K. (1983). Traité fondamental de la foi. Paris: Centurion.

Ricoeur, P. (1986). Du texte à l'action: Essai d'herméneutique II. Paris: Seuil. Savard, P. (1994). Quels types de chrétiens a formés le scoutisme: L'exemple du Canada. Dans G. Cholvy \& M.-T. Cheroutre (Dir.), Le scoutisme (pp. 225-235). Paris: Cerf.

Spence, D.A. (1973). Towards a theology of leisure with special reference to creativity. Ottawa, ON: Canadian Parks and Recreation Association.

Tillich, P. (1957). Dynamics of faith. New York: Harper \& Row.

Tillich, P. (1969). La dimension oubliée. Bruges, Belgique: Desclée de Brouwer.

Tillich, P. (1970). Théologie systématique. Paris: Planète.

Traduction Oecuménique de la Bible. (1988). Paris: Cerf/Société Biblique Française.

Tremblay, P. (1997). On ne sait pas jusqu'à quel point l'éloignement des jeunes nous appauvrit. Revue Notre-Dame, 8, 14-18.

Valadier, P. (1987). L'Église en procès. Paris: Flammarion.

Waaijman, K. (1993). Toward a phenomenological definition of spirituality. Studies in Spirituality, 3, 5-57.

Waaijman, K. (2004). Challenges of spirituality in contemporary times. Spirituality, Lecture Series 3, 1-14.

Waldenfels, H. (1990). Manuel de théologie fondamentale. Paris: Cerf.

Wiesel, E. (1958). La nuit. Paris: Minuit, 1958.

World Organisation of the Scout Movement (2006). World scouting report. Geneva, Switzerland: World Scout Bureau. 